\title{
CuCl-catalyzed aerobic oxidation of 2,3-allenols to 1,2-allenic ketones with 1:1 combination of phenanthroline and bipyridine as ligands
}

\author{
Shuxu Gao ${ }^{1}$, Yu Liu ${ }^{1}$ and Shengming Ma*1,2
}

\section{Full Research Paper}

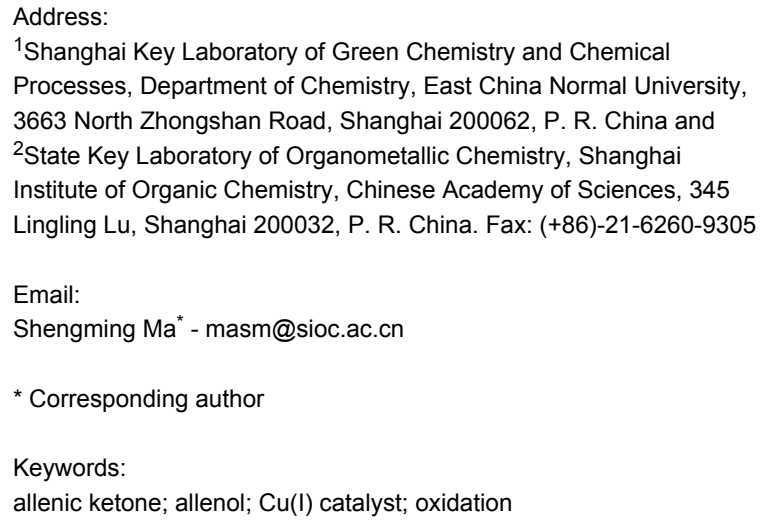

${ }^{1}$ Shanghai Key Laboratory of Green Chemistry and Chemical Processes, Department of Chemistry, East China Normal University, 3663 North Zhongshan Road, Shanghai 200062, P. R. China and ${ }^{2}$ State Key Laboratory of Organometallic Chemistry, Shanghai Institute of Organic Chemistry, Chinese Academy of Sciences, 345 Lingling Lu, Shanghai 200032, P. R. China. Fax: (+86)-21-6260-9305

\section{Email:}

Shengming $\mathrm{Ma}^{*}$ - masm@sioc.ac.cn

* Corresponding author

Keywords:

allenic ketone; allenol; $\mathrm{Cu}(\mathrm{I})$ catalyst; oxidation

Beilstein J. Org. Chem. 2011, 7, 396-403.

doi:10.3762/bjoc.7.51

Received: 25 December 2010

Accepted: 11 March 2011

Published: 07 April 2011

This article is part of the Thematic Series "Allene chemistry".

Guest Editor: K. M. Brummond

(c) 2011 Gao et al; licensee Beilstein-Institut.

License and terms: see end of document.

\begin{abstract}
A protocol has been developed to prepare 1,2-allenyl ketones using molecular oxygen in air or pure oxygen as the oxidant from 2,3allenylic alcohols with moderate to good yields under mild conditions. In this reaction $\mathrm{CuCl}(20 \mathrm{~mol} \%)$ with 1,10-phenanthroline $(10 \mathrm{~mol} \%)$ and bipyridine (10 mol \%) was used as the catalyst. It is interesting to observe that the use of the mixed ligands is important for the higher yields of this transformation: With the monoligand approach developed by Markó et al., the yields are relatively lower.
\end{abstract}

\section{Introduction}

The oxidation of alcohols is one of many fundamental reactions in organic synthesis [1,2]. Usually, stoichiometric oxidants such as $\mathrm{MnO}_{2}$ [3], PCC [4], PDC [4], etc. were employed for this type of transformation. However, the cost and the byproducts derived from these reagents cause economic and environmental problems [5]. In the past decades, much attention has been paid to catalytic oxidation of alcohols using molecular oxygen as the oxidant with $\mathrm{Pd}$ [6-10], $\mathrm{Cu}$ [11-13], $\mathrm{Ru}[14,15]$ as the catalysts.
1,2-Allenic ketones have become very useful in organic synthesis [16-33]. Current methods for the oxidation of allenic alcohols to ketones include oxidation with $\mathrm{MnO}_{2}$ [30,34,35], Swern oxidation [17,24] or Dess-Martin oxidation [16,17,24,25,28,31-33]: Catalytic aerobic oxidation has not so far been reported. Due to the synthetic potential of 1,2-allenyl ketones, it is desirable to develop an aerobic oxidation protocol for 2,3-allenols. In this paper we wish to report the $\mathrm{CuCl}$ - 
catalyzed aerobic oxidation of 2,3-allenols by applying a mixed ligand approach using copper as the catalyst $[12,13]$.

\section{Results and Discussion}

After screening the Pd- [6-10] and Ru-catalyzed [14,15] protocols without success, we began a study of the oxidation of 2-hexyl-1-phenylbuta-2,3-diene-1-ol (1a) with $\mathrm{O}_{2}$ based on the pioneering study of oxidation of normal simple alcohols by Markó et al. [12]. Under the original conditions [12], the expected allenylic ketone $2 \mathrm{a}$ was obtained in $59 \%$ yield when $\mathrm{CuCl}$ and 1,10-phenanthroline were used (Table 1, entry 1). A series of bases and solvents were then screened for the oxidation of 1a. The results are summarized in Table 1 and Table 2. We found that (1) $\mathrm{K}_{2} \mathrm{CO}_{3}$ is the most effective base (Table 1, entry 1) and that organic bases such as $\mathrm{NEt}_{3}$ and DBU are generally ineffective (Table 1, entries 6 and 7); (2) toluene is the best solvent (Table 2).

In order to improve the yield further, we examined the effect of ligands. When 2,2'-bipyridine, which has a weaker coordinating ability, was used [36], the yield of 2a was lower (Table 3, entry 2). With 4,7-diphenyl-1,10-phenanthroline the

Table 1: Screening of bases for the CuCl-catalyzed oxidation of $1 \mathrm{a}^{\mathrm{a}}$.
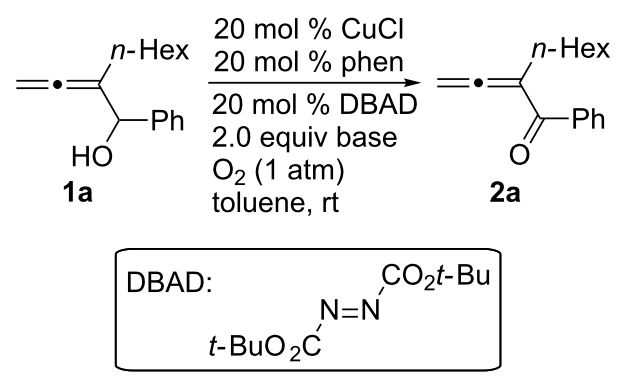

\begin{tabular}{cccc}
\hline entry & base & time (h) & yield of $\mathbf{2 a ~}(\%)^{\mathrm{b}}$ \\
\hline 1 & $\mathrm{~K}_{2} \mathrm{CO}_{3}$ & 40 & $59^{\mathrm{c}, \mathrm{d}}$ \\
2 & $\mathrm{Na}_{2} \mathrm{CO}_{3}$ & 48 & $22^{\mathrm{e}}$ \\
3 & $\mathrm{Cs}_{2} \mathrm{CO}_{3}$ & 35 & $27^{\mathrm{d}, \mathrm{f}, \mathrm{g}}$ \\
4 & $\mathrm{KHCO}_{3}$ & 45 & $15^{\mathrm{h}}$ \\
5 & $\mathrm{KOH}^{\mathrm{i}}$ & 48 & $39^{\mathrm{j}}$ \\
6 & $\mathrm{NEt}_{3}$ & 45 & $\mathrm{NR}^{\mathrm{k}}$ \\
7 & $\mathrm{DBU}$ & 45 & $\mathrm{NR}^{\mathrm{l}}$ \\
\hline
\end{tabular}

aThe reaction was carried out using $0.3 \mathrm{mmol}$ of $1 \mathrm{a}, 20 \mathrm{~mol} \%$ of $\mathrm{CuCl}$, $20 \mathrm{~mol} \%$ of phen, $20 \mathrm{~mol} \%$ of DBAD, and 2.0 equiv of base in $3 \mathrm{~mL}$ of toluene under $1 \mathrm{~atm}$ of oxygen unless otherwise stated. ${ }^{\mathrm{b} 1} \mathrm{H}$ NMR yield using $\mathrm{CH}_{2} \mathrm{Br}_{2}$ as the internal standard. ${ }^{\mathrm{C}} 1.0$ equiv $\mathrm{K}_{2} \mathrm{CO}_{3}$ was used. d Isolated yield. ${ }^{\mathrm{e}} 50 \%$ of $1 \mathrm{a}$ was recovered as determined by ${ }^{1} \mathrm{H}$ NMR analysis. ${ }^{\mathrm{f}} 15 \mathrm{~mol} \%$ of catalyst was used. $932 \%$ of $1 \mathrm{a}$ was recovered by column chromatography. ${ }^{\mathrm{h}} 53 \%$ of $1 \mathrm{a}$ was recovered as determined by ${ }^{1} \mathrm{H}$ NMR analysis. ${ }^{i} 100 \mathrm{mg}$ of $3 \AA \mathrm{MS}$ and $20 \mathrm{~mol} \%$ of $\mathrm{KOH}$ was used. ${ }^{\mathrm{j}} 28 \%$ of $1 \mathrm{a}$ was recovered as determined by ${ }^{1} \mathrm{H}$ NMR analysis. ${ }^{\mathrm{k}} 70 \%$ of $1 \mathrm{a}$ was recovered as determined by ${ }^{1} \mathrm{H}$ NMR analysis. ${ }^{1} 72 \%$ of 1 a was recovered by column chromatography.
Table 2: Screening of solvents for the $\mathrm{CuCl}$-catalyzed oxidation of $1 \mathrm{a}^{\mathrm{a}}$

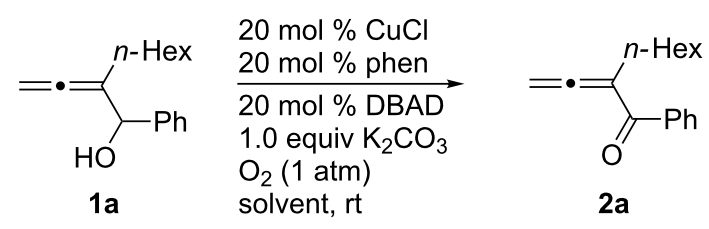

\begin{tabular}{cccc}
\hline entry & solvent & time (h) & yield of $\mathbf{2 a ~}(\%)$ \\
\hline 1 & toluene & 40 & $59^{\mathrm{b}}$ \\
2 & $\mathrm{CH}_{3} \mathrm{CN}$ & 48 & $\mathrm{NR}^{\mathrm{c}}$ \\
3 & $\mathrm{DCE}$ & 47 & $31^{\mathrm{d}}$ \\
4 & $\mathrm{CHCl}_{3}$ & 47 & $20^{\mathrm{e}}$ \\
5 & $\mathrm{DMF}$ & 47 & $\mathrm{NR}^{\mathrm{f}}$ \\
\hline
\end{tabular}

aThe reaction was carried out using $0.3 \mathrm{mmol}$ of $1 \mathrm{a}, 20 \mathrm{~mol} \%$ of $\mathrm{CuCl}$, $20 \mathrm{~mol} \%$ of phen, $20 \mathrm{~mol} \%$ of DBAD, and 1.0 equiv of $\mathrm{K}_{2} \mathrm{CO}_{3}$ in $3 \mathrm{~mL}$ of solvent under $1 \mathrm{~atm}$ of oxygen. ${ }^{\mathrm{b}}$ Isolated yield. ${ }^{\mathrm{c}} 64 \%$ of $1 \mathrm{a}$ was recovered as determined by ${ }^{1} \mathrm{H}$ NMR analysis. ${ }^{\mathrm{d} 1} \mathrm{H}$ NMR yield using $\mathrm{CH}_{2} \mathrm{Br}_{2}$ as the internal standard. ${ }^{\mathrm{e}} 78 \%$ of $1 \mathrm{a}$ was recovered as determined by ${ }^{1} \mathrm{H}$ NMR analysis. ${ }^{\mathrm{f}} 76 \%$ of $1 \mathrm{a}$ was recovered as determined by ${ }^{1} \mathrm{H}$ NMR analysis.

yield was slightly improved to $66 \%$ (Table 3 , entry 3 ). These experimental results obviously indicated that the $\mathrm{CuCl}-$ catalyzed oxidation of allenic alcohol was influenced by the coordinating ability of nitrogen ligands. Consequently, we carried out the reaction with a mixture of a stronger coordinating ligand together with a relatively weaker coordinating ligand. Indeed, it was interesting to observe that when 4,7diphenyl-1,10-phenanthroline and 2,2'-bipyridine were mixed in the ratio of $1: 1[37,38]$, the isolated yield was improved to $82 \%$ (Table 3, entry 6). The yield with 1,10-phenanthroline and 2,2'bipyridine (1:1) was $83 \%$ (Table 3 , entry 10 ). However, 4,7 diphenyl-1,10-phenanthroline is relatively expensive ( $1 \mathrm{~g}, \$ 94$, Aldrich), so the cheaper 1,10-phenanthroline (5 g, \$26.4, Aldrich) was used for further study. The effect of ratio of 1,10phenanthroline vs 2,2'-bipyridine on the yield was also studied: A ratio of 1:1 proved to be the best (Table 3, entries 7-12). This may be explained by considering that the coordination of $2,2^{\prime}-$ bipyridine is important for the formation of the catalytically active species and may be easily replaced with that of the alcohol. We also tried $N$-methylimidazole (NMI), which was used in oxidation of primary aliphatic alcohols reported by Markó et al. [13], however, both the turnover and yield were low (Table 3, entry 14). Both 2,9-dimethyl-4,7-diphenyl-1,10phenanthroline and iPr-Pybox were ineffective in this reaction (Table 3, entries 4 and 5).

Some other $\mathrm{Cu}(\mathrm{I})$ catalysts, such as $\mathrm{CuBr}, \mathrm{CuI}$, and $\mathrm{CuCN}$ were also investigated, but no higher yield was achieved (Table 4). Further studies led to the observation that air (300 psi, $35^{\circ} \mathrm{C}$ (oil bath)) could be used instead of pure oxygen 
Table 3: Screening for different nitrogen ligands in the CuCl-catalyzed oxidation of $1 \mathrm{a}^{\mathrm{a}}$.

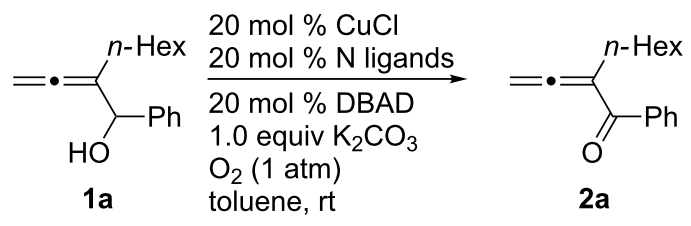

\begin{tabular}{|c|c|c|c|c|}
\hline entry & ligand $1(\mathrm{~mol} \%)$ & ligand $2(\mathrm{~mol} \%)$ & time $(\mathrm{h})$ & yield of $2 a(\%)^{b}$ \\
\hline 1 & & - & 40 & 61 \\
\hline 2 & & - & 46 & $43^{c}$ \\
\hline 3 & & - & 14.5 & 66 \\
\hline 4 & & - & 45 & $N R^{d}$ \\
\hline 5 & & - & 24 & $N R^{e}$ \\
\hline 6 & & L-B (10) & 45.5 & 82 \\
\hline & & & & \\
\hline 7 & L-A (17.5) & L-B (2.5) & 40 & 79 \\
\hline 8 & L-A (15.0) & L-B (5.0) & 40 & 65 \\
\hline 9 & L-A (12.5) & L-B (7.5) & 40 & 82 \\
\hline 10 & L-A (10.0) & L-B (10.0) & 42 & 83 \\
\hline 11 & L-A (7.5) & L-B (12.5) & 42 & 78 \\
\hline 12 & L-A (5.0) & L-B (15.0) & 40 & 73 \\
\hline 13 & L-A (2.5) & L-B (17.5) & 40 & 68 \\
\hline $14^{f}$ & L-A (5.0) & NMI (7.0) & 35 & 17 \\
\hline
\end{tabular}

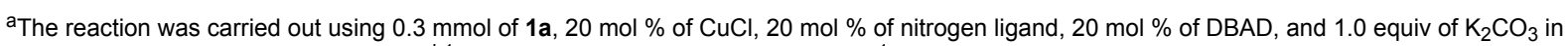
$3 \mathrm{~mL}$ of toluene under $1 \mathrm{~atm}$ of oxygen. ${ }^{\mathrm{b} 1} \mathrm{H}$ NMR yields determined by $300 \mathrm{MHz},{ }^{1} \mathrm{H}$ NMR analysis using $\mathrm{CH}_{2} \mathrm{Br}_{2}$ as the internal standard. ${ }^{c} 52 \%$ of $1 \mathrm{a}$ was recovered as determined by ${ }^{1} \mathrm{H}$ NMR analysis. ${ }^{d} 87 \%$ of $1 \mathrm{a}$ was recovered as determined by ${ }^{1} \mathrm{H}$ NMR analysis. ${ }^{e} 82 \%$ of $1 \mathrm{a}$ was recovered as determined by ${ }^{1} \mathrm{H}$ NMR analysis. ${ }^{\mathrm{f}}$ The reaction was carried out using $0.5 \mathrm{mmol}$ of $1 \mathrm{a}, 5 \mathrm{~mol} \%$ of CuCl, $5 \mathrm{~mol} \%$ of $t$-BuOK, $5 \mathrm{~mol} \%$ of DBAD and the indicated ligands in $5 \mathrm{~mL}$ of $\mathrm{C}_{6} \mathrm{H}_{5} \mathrm{~F}$ at $70{ }^{\circ} \mathrm{C}$ under $1 \mathrm{~atm}$ of oxygen. $52 \%$ of $1 \mathrm{a}$ was recovered as determined by ${ }^{1} \mathrm{H}$ NMR analysis.

(1 atm, $\left.15-24{ }^{\circ} \mathrm{C}\right)$ to shorten the reaction time from 40 to 10 hours and the yield was similar (86\%) (Table 5, entry 1 ). Thus, $20 \mathrm{~mol} \%$ of $\mathrm{CuCl}, 10 \mathrm{~mol} \%$ of 1,10-phenanthroline,
$10 \mathrm{~mol} \%$ of 2,2'-bipyridine and $50 \mathrm{~mol} \%$ of $\mathrm{K}_{2} \mathrm{CO}_{3}$ in toluene with air $\left(300 \mathrm{psi}, 35^{\circ} \mathrm{C}\right)$ as the oxidant were defined as the standard conditions. 
Under the standard conditions a series of 1-aryl-2,3-allenols were oxidized to the corresponding 1,2-allenic aryl ketones: A para-nitro group led to a $63 \%$ yield of $2 \mathbf{e}$ (Table 5, entry 5); heteroaryl groups such as furanyl and thienyl were also tolerated under the reaction conditions, affording the corresponding allenic ketones $\mathbf{2 f}$ and $\mathbf{2 g}$ in $61 \%$ and $73 \%$ yields, respectively (Table 5, entries 6 and 7), whilst the reaction of 1-naphthylsubstituted $\mathbf{1 h}$ afforded $\mathbf{2 h}$ in $74 \%$ yield (Table 5 , entry 8 ). Trisubstituted allenic alcohol $\mathbf{1} \mathbf{j}$ was also oxidized to the corresponding allenic ketone $\mathbf{2} \mathbf{j}$ in $91 \%$ yield (Table 5, entry 10 ).

Table 4: Screening of other $\mathrm{Cu}(\mathrm{I})$ sources.

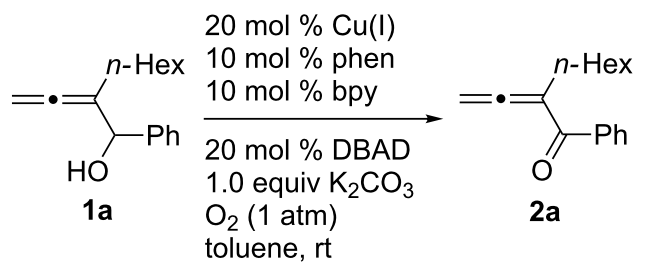

\begin{tabular}{cccc}
\hline entry & $\mathrm{Cu}(\mathrm{l})$ & time (h) & isolated yield of 2a \\
\hline 1 & $\mathrm{CuBr}$ & 40 & $68 \%$ \\
2 & $\mathrm{Cul}$ & 40 & $49 \%$ \\
3 & $\mathrm{CuCN}$ & 41 & $45 \%$
\end{tabular}

The reaction may be easily carried out on a $1 \mathrm{~g}$ scale: the oxidation of allenol $\mathbf{1 k}$ afforded the corresponding allenic ketone $\mathbf{2 k}$ in $74 \%$ yield in 12 hours with just $10 \mathrm{~mol} \%$ of $\mathrm{CuCl}$ and $5 \mathrm{~mol} \%$ each of 1,10-phenanthroline and 2,2'-bipyridine (Scheme 1).

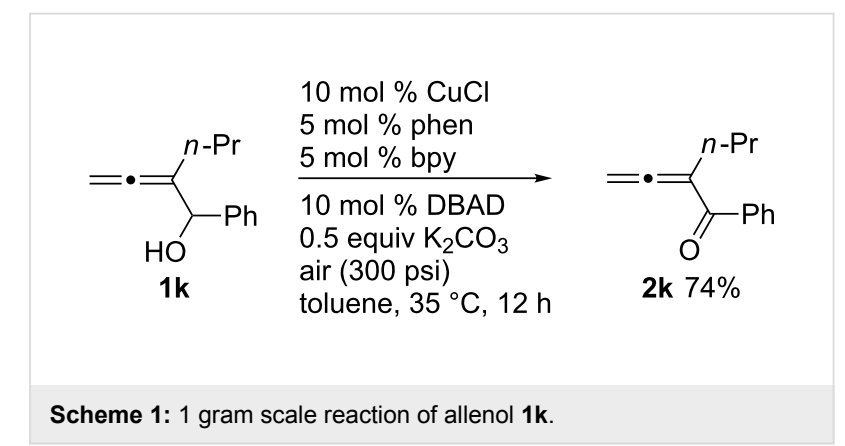

When the reaction of 1-alkyl-substituted-2,3-allenols oxidation was conducted under $1 \mathrm{~atm}$ of oxygen at $60{ }^{\circ} \mathrm{C}, 81 \%$ of conversion was observed and the corresponding allenic ketones $\mathbf{2 l}$ and $2 \mathrm{~m}$ were obtained in $58 \%$ and $60 \%$ isolated yields $(72 \%$ and $74 \%$ based on the starting material consumed), respectively (Scheme 2). As a comparison, it should be noted that when $\mathbf{1 l}$ was oxidized with air $\left(300 \mathrm{psi}, 60^{\circ} \mathrm{C}\right)$, the allenic ketone $\mathbf{2 l}$ was formed in $43 \%{ }^{1} \mathrm{H}$ NMR yield with $73 \%$ conversion of $\mathbf{1 l}$ within 10 hours.

Table 5: The CuCl-catalyzed oxidation of allenic alcohols using air as the oxidant ${ }^{\text {a }}$.

\begin{tabular}{|c|c|c|c|c|c|}
\hline \multirow{3}{*}{ entry } & \multirow[t]{2}{*}{$\mathrm{R}^{3}$} & \multicolumn{2}{|c|}{$\begin{array}{l}20 \mathrm{~mol} \% \mathrm{CuCl} \\
10 \mathrm{~mol} \% \text { phen } \\
10 \mathrm{~mol} \% \text { bpy } \\
20 \mathrm{~mol} \% \mathrm{DBAD}^{\circ} \\
0.5 \text { equiv } \mathrm{K}_{2} \mathrm{CO}_{3} \\
\text { air ( } 300 \mathrm{psi}) \\
\text { toluene, } 35^{\circ} \mathrm{C}\end{array}$} & \multirow{3}{*}{ time (h) } & \multirow{3}{*}{ yield $(\%)^{b}$} \\
\hline & & \multicolumn{2}{|l|}{ substrate } & & \\
\hline & $\mathrm{R}^{1}$ & $\mathrm{R}^{2}$ & $\mathrm{R}^{3}$ & & \\
\hline 1 & $\mathrm{Ph}$ & $n-\mathrm{C}_{6} \mathrm{H}_{13}$ & $H(1 a)$ & 10 & $86(2 a)$ \\
\hline 2 & $p-\mathrm{EtC}_{6} \mathrm{H}_{4}$ & $n-\operatorname{Pr}$ & $H(1 b)$ & 10 & $83(2 b)$ \\
\hline 3 & $p-\mathrm{BrC}_{6} \mathrm{H}_{4}$ & $n-\operatorname{Pr}$ & $H(1 c)$ & 6 & $80(2 c)$ \\
\hline 4 & $p-\mathrm{ClC}_{6} \mathrm{H}_{4}$ & $n-\mathrm{C}_{6} \mathrm{H}_{13}$ & $H(1 d)$ & 6 & $78(2 d)$ \\
\hline 5 & $p-\mathrm{O}_{2} \mathrm{NC}_{6} \mathrm{H}_{4}$ & $n-\mathrm{C}_{6} \mathrm{H}_{13}$ & $\mathrm{H}(1 \mathrm{e})$ & 6 & $63(2 e)$ \\
\hline 6 & 3-furanyl & $n-\mathrm{C}_{6} \mathrm{H}_{13}$ & $H(\mathbf{1 f})$ & 11 & $61(2 f)$ \\
\hline 7 & 3-thienyl & $n-\mathrm{C}_{5} \mathrm{H}_{11}$ & $H(1 \mathbf{g})$ & 8.5 & $73(\mathbf{2 g})$ \\
\hline 8 & 1-naphthyl & $\mathrm{Me}$ & $H(1 h)$ & 11 & $74(2 h)$ \\
\hline 9 & $\mathrm{Ph}$ & allyl & $H(1 i)$ & 11 & $75(2 \mathbf{i})$ \\
\hline 10 & $\mathrm{Ph}$ & $\mathrm{Bu}$ & $n-\mathrm{C}_{5} \mathrm{H}_{11}(\mathbf{1} \mathbf{j})$ & 11 & 91 (2j) \\
\hline
\end{tabular}

aThe reaction was carried out using $0.3 \mathrm{mmol}$ of $1,20 \mathrm{~mol} \%$ of $\mathrm{CuCl}, 10 \mathrm{~mol} \%$ of phen, $10 \mathrm{~mol} \%$ of bpy, $20 \mathrm{~mol} \%$ of DBAD, and 0.5 equiv of

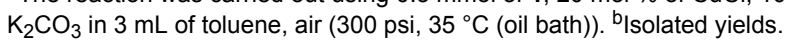




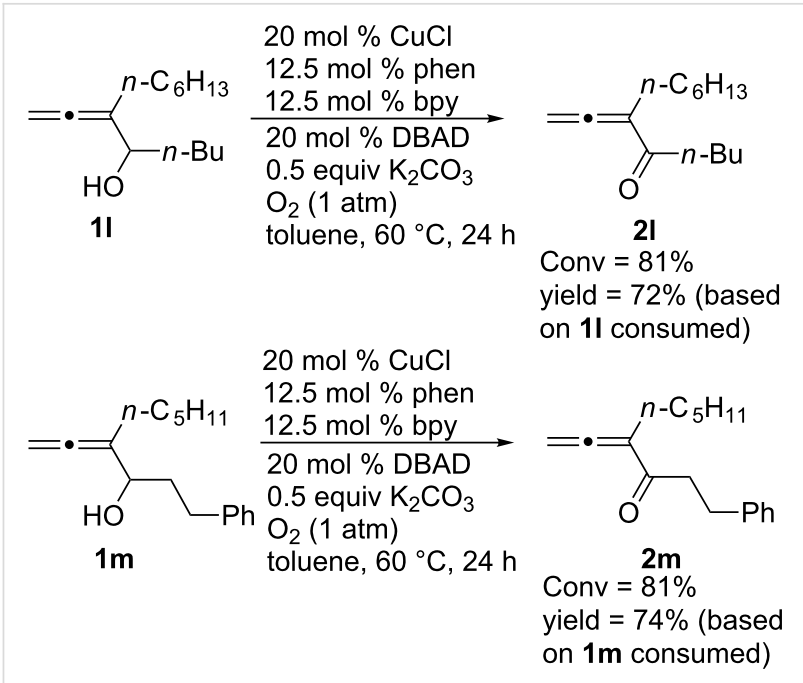

Scheme 2: The oxidation of $1 \mathrm{l}$ and $1 \mathrm{~m}$ under $1 \mathrm{~atm}$ of oxygen.

\section{Conclusion}

In conclusion, we have developed a method for the aerobic oxidation of 2,3-allenols, which uses molecular oxygen in air or pure oxygen as the oxidant. In this reaction, $\mathrm{CuCl}$ with a $1: 1$ ratio of 1,10-phenanthroline and bipyridine was used as the catalyst to provide the best results. A series of 1,2-allenic ketones were obtained in moderate to good yields under mild conditions. Compared to the traditional monoligand approach, allenols are obviously unique demanding a mixed ligands approach for better yields probably as a consequence of the coordinating ability of the allene moiety. Further study in this area is being pursued in this laboratory.

\section{Experimental}

\section{General experimental methods for starting materials}

The starting allenols $\mathbf{1 a}-\mathbf{e}, \mathbf{1} \mathbf{i}, \mathbf{1 k}, \mathbf{1}, \mathbf{1} \mathbf{m}$ were prepared via the reaction of propargyl bromides and corresponding aldehydes in the presence of $\mathrm{SnCl}_{2}$ and $\mathrm{NaI}$ in DMF [39,40]; allenols 1f [41], $\mathbf{1 g}$ [42], $\mathbf{1 h}$ [43], and $\mathbf{1 j}$ [44] were prepared as reported. These starting allenols were purified by flash chromatography before use.

\section{General experimental procedure for the aerobic oxidation of allenic alcohols \\ 2-Hexyl-1-phenylbuta-2,3-dien-1-one (2a)}

Typical procedure: 1,10 -phenanthroline $(5.5 \mathrm{mg}, 0.03 \mathrm{mmol})$, 2,2'-bipyridine $(4.7 \mathrm{mg}, 0.03 \mathrm{mmol}), \mathrm{CuCl}(5.9 \mathrm{mg}$, $0.06 \mathrm{mmol}), \mathrm{K}_{2} \mathrm{CO}_{3}(20.6 \mathrm{mg}, 0.15 \mathrm{mmol}$ ), and $1.5 \mathrm{~mL}$ of dry toluene were added successively into an oven dried reaction vessel (sealed with a stopper to isolate the contents from atmospheric moisture). The resulting mixture was stirred at $\mathrm{rt}$ for $0.5 \mathrm{~h}$. Then the stopper was removed to add DBAD (13.7 mg,
$0.06 \mathrm{mmol}), 2$-hexyl-1-phenylbuta-2,3-dien-1-ol (69.6 mg, $0.3 \mathrm{mmol}$ ), and $1.5 \mathrm{~mL}$ of dry toluene. The reaction vessel was then transferred to an autoclave, which was charged with air to a pressure of $300 \mathrm{psi}$, and stirred at $35^{\circ} \mathrm{C}$ (oil bath). After $10 \mathrm{~h}$, the pressure was carefully released in the hood, the mixture filtered through a short column of silica gel (100-140 mesh) and washed with diethyl ether. Evaporation of the solvent and flash chromatography on silica gel (eluent: petroleum ether/ diethyl ether $=30: 1)$ afforded 2a $(59.3 \mathrm{mg}, 86 \%)$ : liquid; ${ }^{1} \mathrm{H}$ NMR (300 MHz, $\left.\mathrm{CDCl}_{3}\right) \delta 7.76(\mathrm{~d}, J=7.2 \mathrm{~Hz}, 2 \mathrm{H}), 7.49(\mathrm{t}$, $J=7.4 \mathrm{~Hz}, 1 \mathrm{H}), 7.39(\mathrm{t}, J=7.2 \mathrm{~Hz}, 2 \mathrm{H}), 5.04(\mathrm{t}, J=2.7 \mathrm{~Hz}$, $2 \mathrm{H}), 2.46-2.35(\mathrm{~m}, 2 \mathrm{H}), 1.58-1.45(\mathrm{~m}, 2 \mathrm{H}), 1.45-1.20(\mathrm{~m}, 6 \mathrm{H})$, $0.89(\mathrm{t}, J=6.6 \mathrm{~Hz}, 3 \mathrm{H}) \mathrm{ppm} ;{ }^{13} \mathrm{C} \mathrm{NMR}\left(75.4 \mathrm{MHz}, \mathrm{CDCl}_{3}\right)$ $\delta 217.0,194.9,138.3,131.9,129.0,127.8,106.9,79.3,31.6$, 28.9, 27.8, 22.6, 14.0 ppm; MS (m/z) $228\left(\mathrm{M}^{+}, 7.25\right), 105$ (100); IR (neat) 2927, 2857, 1933, 1653, 1599, 1450, 1312, $1273 \mathrm{~cm}^{-1}$; HRMS-EI $(\mathrm{m} / \mathrm{z})$ calcd for $\mathrm{C}_{16} \mathrm{H}_{20} \mathrm{O}^{+}\left[\mathrm{M}^{+}\right]$: 228.1514 ; found: 228.1517 .

\section{2-Propyl-1-(4-ethylphenyl)buta-2,3-dien-1-one (2b)}

The reaction of 1,10-phenanthroline ( $5.3 \mathrm{mg}, 0.03 \mathrm{mmol}), 2,2^{\prime}-$ bipyridine ( $4.6 \mathrm{mg}, 0.03 \mathrm{mmol}), \mathrm{CuCl}(5.9 \mathrm{mg}, 0.06 \mathrm{mmol})$, $\mathrm{K}_{2} \mathrm{CO}_{3}(20.8 \mathrm{mg}, 0.15 \mathrm{mmol})$, dry toluene $(1.5 \mathrm{~mL})$, DBAD (13.7 mg, $0.06 \mathrm{mmol}$ ), and 2-propyl-1-(4-ethylphenyl)buta-2,3dien-1-ol (64.7 mg, $0.3 \mathrm{mmol}) /$ dry toluene $(1.5 \mathrm{~mL})$ afforded $\mathbf{2 b}$ (53.0 mg, 83\%): liquid; ${ }^{1} \mathrm{H} \mathrm{NMR}\left(300 \mathrm{MHz}, \mathrm{CDCl}_{3}\right) \delta 7.73$ (d, $J=8.1 \mathrm{~Hz}, 2 \mathrm{H}), 7.22(\mathrm{~d}, J=8.1 \mathrm{~Hz}, 2 \mathrm{H}), 5.05(\mathrm{t}, J=2.9 \mathrm{~Hz}$, $2 \mathrm{H}), 2.69$ (q, $J=7.6 \mathrm{~Hz}, 2 \mathrm{H}), 2.44-2.32(\mathrm{~m}, 2 \mathrm{H}), 1.63-1.45$ (m, $2 \mathrm{H}), 1.25$ (t, $J=7.5 \mathrm{~Hz}, 3 \mathrm{H}), 0.99$ (t, $J=7.4 \mathrm{~Hz}, 3 \mathrm{H}) \mathrm{ppm}$; ${ }^{13} \mathrm{C}$ NMR (75.4 MHz, $\left.\mathrm{CDCl}_{3}\right) \delta 216.5,194.4,148.8,135.8$, 129.3, 127.3, 106.5, 79.1, 30.1, 28.8, 21.1, 15.1, 13.7 ppm; MS $(\mathrm{m} / \mathrm{z}) 214\left(\mathrm{M}^{+}, 1.75\right), 133$ (100); IR (neat) 2964, 2931, 2872, 1933, 1650, 1607, 1458, 1414, 1273, 1182, $1058 \mathrm{~cm}^{-1}$; HRMSEI $(m / z)$ calcd for $\mathrm{C}_{15} \mathrm{H}_{18} \mathrm{O}^{+}\left[\mathrm{M}^{+}\right]$: 214.1358; found: 214.1360 .

\section{2-Propyl-1-(4-bromophenyl)buta-2,3-dien-1-one (2c)} The reaction of 1,10-phenanthroline ( $5.4 \mathrm{mg}, 0.03 \mathrm{mmol}), 2,2^{\prime}-$ bipyridine ( $4.6 \mathrm{mg}, 0.03 \mathrm{mmol}), \mathrm{CuCl}(5.9 \mathrm{mg}, 0.06 \mathrm{mmol})$, $\mathrm{K}_{2} \mathrm{CO}_{3}(20.6 \mathrm{mg}, 0.15 \mathrm{mmol})$, dry toluene $(1.5 \mathrm{~mL})$, DBAD (13.8 $\mathrm{mg}, 0.06 \mathrm{mmol}$ ), and 2-propyl-1-(4-bromophenyl)buta2,3-dien-1-ol $(80.3 \mathrm{mg}, 0.3 \mathrm{mmol}) /$ dry toluene $(1.5 \mathrm{~mL})$ afforded 2c (64.1 mg, 80\%): liquid; ${ }^{1} \mathrm{H}$ NMR (300 MHz, $\left.\mathrm{CDCl}_{3}\right) \delta 7.62(\mathrm{~d}, J=8.7 \mathrm{~Hz}, 2 \mathrm{H}), 7.52(\mathrm{~d}, J=8.4 \mathrm{~Hz}, 2 \mathrm{H})$, $5.07(\mathrm{t}, J=2.9 \mathrm{~Hz}, 2 \mathrm{H}), 2.41-2.30(\mathrm{~m}, 2 \mathrm{H}), 1.60-1.45(\mathrm{~m}, 2 \mathrm{H})$, 0.97 (t, $J=7.5 \mathrm{~Hz}, 3 \mathrm{H}) \mathrm{ppm} ;{ }^{13} \mathrm{C} \mathrm{NMR}\left(75.4 \mathrm{MHz}, \mathrm{CDCl}_{3}\right)$ $\delta 217.0,193.7,137.0,131.1,130.5,126.7,106.7,79.7,29.7$, 21.1, $13.7 \mathrm{ppm}$; MS $(\mathrm{m} / \mathrm{z}) 266\left(\mathrm{M}^{+}\left({ }^{81} \mathrm{Br}\right), 1.68\right), 264\left(\mathrm{M}^{+}\right.$ $\left.\left({ }^{79} \mathrm{Br}\right), 1.76\right), 185$ (100); IR (neat) 2961, 2929, 2870, 1931, $1653,1585,1458,1391,1270,1071,1010 \mathrm{~cm}^{-1}$; HRMS-EI $(\mathrm{m} / z)$ calcd for $\mathrm{C}_{13} \mathrm{H}_{13} \mathrm{O}^{81} \mathrm{Br}^{+}\left[\mathrm{M}^{+}\right]$: 266.0129; found: 266.0136 . 


\section{2-Hexyl-1-(4-chlorophenyl)buta-2,3-dien-1-one (2d)}

The reaction of 1,10-phenanthroline ( $5.5 \mathrm{mg}, 0.03 \mathrm{mmol}), 2,2^{\prime}$ bipyridine ( $4.8 \mathrm{mg}, 0.03 \mathrm{mmol}), \mathrm{CuCl}(6.0 \mathrm{mg}, 0.06 \mathrm{mmol})$, $\mathrm{K}_{2} \mathrm{CO}_{3}(20.9 \mathrm{mg}, 0.15 \mathrm{mmol})$, dry toluene $(1.5 \mathrm{~mL})$, DBAD (13.8 mg, $0.06 \mathrm{mmol})$, and 2-hexyl-1-(4chlorophenyl)buta-2,3-dien-1-ol (79.6 mg, $0.3 \mathrm{mmol}) /$ dry toluene $(1.5 \mathrm{~mL})$ afforded $\mathbf{2 d}(62.0 \mathrm{mg}, 78 \%)$ : liquid; ${ }^{1} \mathrm{H} \mathrm{NMR}$ $\left(300 \mathrm{MHz}, \mathrm{CDCl}_{3}\right) \delta 7.70(\mathrm{~d}, J=8.4 \mathrm{~Hz}, 2 \mathrm{H}), 7.35(\mathrm{~d}, J=8.4$ $\mathrm{Hz}, 2 \mathrm{H}), 5.06(\mathrm{t}, J=2.6 \mathrm{~Hz}, 2 \mathrm{H}), 2.43-2.32(\mathrm{~m}, 2 \mathrm{H}), 1.55-1.42$ $(\mathrm{m}, 2 \mathrm{H}), 1.43-1.18(\mathrm{~m}, 6 \mathrm{H}), 0.88(\mathrm{t}, J=6.3 \mathrm{~Hz}, 3 \mathrm{H}) \mathrm{ppm}$; ${ }^{13} \mathrm{C} \mathrm{NMR}\left(75.4 \mathrm{MHz}, \mathrm{CDCl}_{3}\right) \delta 217.0,193.6,138.2,136.6$, 130.4, 128.1, 106.9, 79.6, 31.6, 28.9, 27.7, 22.5, 14.0 ppm; MS $(\mathrm{m} / \mathrm{z}) 264\left(\mathrm{M}^{+}\left({ }^{37} \mathrm{Cl}\right), 0.76\right), 262\left(\mathrm{M}^{+}\left({ }^{35} \mathrm{Cl}\right), 2.08\right), 139(100)$; IR (neat) 2927, 2857, 1931, 1654, 1590, 1460, 1397, 1274, $1091 \mathrm{~cm}^{-1}$; HRMS-EI $(\mathrm{m} / \mathrm{z})$ calcd for $\mathrm{C}_{16} \mathrm{H}_{19} \mathrm{O}^{35} \mathrm{Cl}^{+}\left[\mathrm{M}^{+}\right]$: 262.1124; found: 262.1130 .

\section{2-Hexyl-1-(4'-nitrophenyl)buta-2,3-dien-1-one (2e)}

The reaction of 1,10-phenanthroline $(5.5 \mathrm{mg}, 0.03 \mathrm{mmol}), 2,2^{\prime}-$ bipyridine (4.7 mg, $0.03 \mathrm{mmol}), \mathrm{CuCl}(5.9 \mathrm{mg}, 0.06 \mathrm{mmol})$, $\mathrm{K}_{2} \mathrm{CO}_{3}(20.8 \mathrm{mg}, 0.15 \mathrm{mmol})$, dry toluene $(1.5 \mathrm{~mL})$, DBAD (13.9 mg, $0.06 \mathrm{mmol}$ ), and 2-hexyl-1-(4-nitrophenyl)buta-2,3dien-1-ol ( $82.7 \mathrm{mg}, 0.3 \mathrm{mmol})$ /dry toluene $(1.5 \mathrm{~mL})$ afforded $2 \mathrm{e}$ $(51.4 \mathrm{mg}, 63 \%)$ (eluent: petroleum ether/diethyl ether $=20: 1)$ : liquid; ${ }^{1} \mathrm{H}$ NMR (300 MHz, $\left.\mathrm{CDCl}_{3}\right) \delta 8.24(\mathrm{~d}, J=8.7 \mathrm{~Hz}, 2 \mathrm{H})$, $7.84(\mathrm{~d}, J=8.7 \mathrm{~Hz}, 2 \mathrm{H}), 5.12$ (t, $J=2.9 \mathrm{~Hz}, 2 \mathrm{H}), 2.44-2.33$ (m, $2 \mathrm{H}), 1.57-1.44(\mathrm{~m}, 2 \mathrm{H}), 1.44-1.20(\mathrm{~m}, 6 \mathrm{H}), 0.89(\mathrm{t}, J=6.5 \mathrm{~Hz}$, $3 \mathrm{H}) \mathrm{ppm} ;{ }^{13} \mathrm{C} \mathrm{NMR}\left(75.4 \mathrm{MHz}, \mathrm{CDCl}_{3}\right) \delta 218.0,193.4,149.4$, 143.6, 129.7, 123.1, 107.6, 80.4, 31.5, 28.8, 27.7, 27.3, 22.5, $14.0 \mathrm{ppm}$; MS (m/z) $273\left(\mathrm{M}^{+}, 2.56\right), 150$ (100); IR (neat) 2927, 2857, 1930, 1660, 1602, 1526, 1461, 1349, 1272, 1104, $1011 \mathrm{~cm}^{-1}$; HRMS-EI $(\mathrm{m} / \mathrm{z})$ calcd for $\mathrm{C}_{16} \mathrm{H}_{19} \mathrm{NO}_{3}{ }^{+}\left[\mathrm{M}^{+}\right]$: 273.1365; found: 273.1367 .

\section{2-Hexyl-1-(3-furanyl)buta-2,3-dien-1-one (2f)}

The reaction of 1,10-phenanthroline (5.4 mg, $0.03 \mathrm{mmol}), 2,2$ bipyridine ( $4.8 \mathrm{mg}, 0.03 \mathrm{mmol}), \mathrm{CuCl}(6.2 \mathrm{mg}, 0.06 \mathrm{mmol})$, $\mathrm{K}_{2} \mathrm{CO}_{3}(21.3 \mathrm{mg}, 0.15 \mathrm{mmol})$, dry toluene $(1.5 \mathrm{~mL})$, DBAD (13.6 mg, $0.06 \mathrm{mmol}$ ), and 2-hexyl-1-(3-furanyl)buta-2,3-dien1-ol $(66.2 \mathrm{mg}, 0.3 \mathrm{mmol}) /$ dry toluene $(1.5 \mathrm{~mL})$ afforded 2f (40.4 mg, 61\%): liquid; ${ }^{1} \mathrm{H}$ NMR (300 MHz, $\left.\mathrm{CDCl}_{3}\right) \delta 8.10$ (s, 1H), $7.37(\mathrm{~s}, 1 \mathrm{H}), 6.81(\mathrm{~d}, J=1.2 \mathrm{~Hz}, 1 \mathrm{H}), 5.21(\mathrm{t}, J=$ $2.9 \mathrm{~Hz}, 2 \mathrm{H}), 2.39-2.27$ (m, $2 \mathrm{H}), 1.52-1.38(\mathrm{~m}, 2 \mathrm{H}), 1.38-1.18$ $(\mathrm{m}, 6 \mathrm{H}), 0.87(\mathrm{t}, J=6.5 \mathrm{~Hz}, 3 \mathrm{H}) \mathrm{ppm} ;{ }^{13} \mathrm{C} \mathrm{NMR}(75.4 \mathrm{MHz}$, $\left.\mathrm{CDCl}_{3}\right) \delta 215.8,186.4,147.4,143.0,126.7,110.0,108.1$, 80.0, 31.6, 28.9, 27.8, 27.6, 22.6, $14.1 \mathrm{ppm}$; MS (m/z) $218\left(\mathrm{M}^{+}\right.$, 3.49), 95 (100); IR (neat) 2956, 2928, 2857, 1933, 1724, 1645, 1561, 1509, 1458, 1379, 1311, 1163, 1077, $1009 \mathrm{~cm}^{-1}$; HRMS-EI $(\mathrm{m} / \mathrm{z})$ calcd for $\mathrm{C}_{14} \mathrm{H}_{18} \mathrm{O}_{2}{ }^{+}\left[\mathrm{M}^{+}\right]$: 218.1307; found: 218.1305 .

\section{2-Pentyl-1-(3-thienyl)buta-2,3-dien-1-one (2g)}

The reaction of 1,10-phenanthroline ( $5.5 \mathrm{mg}, 0.03 \mathrm{mmol}), 2,2^{\prime}-$ bipyridine ( $4.8 \mathrm{mg}, 0.03 \mathrm{mmol}), \mathrm{CuCl}$ (5.9 mg, $0.06 \mathrm{mmol}$ ), $\mathrm{K}_{2} \mathrm{CO}_{3}(20.9 \mathrm{mg}, 0.15 \mathrm{mmol})$, dry toluene $(1.5 \mathrm{~mL})$, DBAD (13.7 mg, $0.06 \mathrm{mmol}$ ), and 2-pentyl-1-(3-thienyl)buta-2,3-dien1-ol (66.4 mg, $0.3 \mathrm{mmol}) /$ dry toluene $(1.5 \mathrm{~mL})$ afforded $\mathbf{2 g}$ $(48.3 \mathrm{mg}, 73 \%)$ (eluent: petroleum ether/diethyl ether $=50: 1)$ : liquid; ${ }^{1} \mathrm{H}$ NMR $\left(300 \mathrm{MHz}, \mathrm{CDCl}_{3}\right) \delta 8.07(\mathrm{~d}, J=1.8 \mathrm{~Hz}, 1 \mathrm{H})$ $7.53(\mathrm{~d}, J=4.8 \mathrm{~Hz}, 1 \mathrm{H}), 7.25\left(\mathrm{dd}, J_{1}=4.8 \mathrm{~Hz}, J_{2}=3.3 \mathrm{~Hz}\right.$, $1 \mathrm{H}), 5.16(\mathrm{~d}, J=2.7 \mathrm{~Hz}, 2 \mathrm{H}), 2.41-2.32(\mathrm{~m}, 2 \mathrm{H}), 1.56-1.42(\mathrm{~m}$, $2 \mathrm{H}), 1.42-1.24(\mathrm{~m}, 4 \mathrm{H}), 0.90(\mathrm{t}, J=6.6 \mathrm{~Hz}, 3 \mathrm{H}) \mathrm{ppm}$; ${ }^{13} \mathrm{C}$ NMR (75.4 MHz, $\left.\mathrm{CDCl}_{3}\right) \delta 216.0,187.1,141.5,132.2$, 128.2, 125.1, 107.6, 79.6, 31.4, 27.9, 27.5, 22.4, 14.0 ppm; MS $(\mathrm{m} / \mathrm{z}) 220\left(\mathrm{M}^{+}, 3.02\right), 111$ (100); IR (neat) 2956, 2927, 2861, 1933, 1641, 1511, 1460, 1411, 1260, $1082 \mathrm{~cm}^{-1}$; HRMS-EI $\left(\mathrm{m} / \mathrm{z}\right.$ ) calcd for $\mathrm{C}_{13} \mathrm{H}_{16} \mathrm{OS}^{+}\left[\mathrm{M}^{+}\right]$: 220.0922; found: 220.0922 .

\section{2-Methyl-1-(1-naphthyl)buta-2,3-dien-1-one (2h)}

The reaction of 1,10-phenanthroline $(5.5 \mathrm{mg}, 0.03 \mathrm{mmol}), 2,2^{\prime}-$ bipyridine ( $4.8 \mathrm{mg}, 0.03 \mathrm{mmol}), \mathrm{CuCl}(6.2 \mathrm{mg}, 0.06 \mathrm{mmol})$, $\mathrm{K}_{2} \mathrm{CO}_{3}(21.4 \mathrm{mg}, 0.15 \mathrm{mmol})$, dry toluene $(1.5 \mathrm{~mL})$, DBAD (13.5 mg, $0.06 \mathrm{mmol}$ ), and 2-methyl-1-naphthylbuta-2,3-dien-1ol $(63.6 \mathrm{mg}, 0.3 \mathrm{mmol}) / \mathrm{dry}$ toluene $(1.5 \mathrm{~mL})$ afforded $\mathbf{2 h}$ (47.2 mg, 74\%, an unknown substance could not be separated via column chromatography and the purity of $\mathbf{2 h}$ is $95 \%$, which was determined by ${ }^{1} \mathrm{H}$ NMR with mesitylene as the internal standard): liquid; ${ }^{1} \mathrm{H} \mathrm{NMR}\left(300 \mathrm{MHz}, \mathrm{CDCl}_{3}\right) \delta 8.11-8.03(\mathrm{~m}$, 1H), 7.95-7.81 (m, 2H), 7.61-7.39 (m, 4H), 4.80 (q, $J=2.8 \mathrm{~Hz}$, 2H), 2.11 (t, $J=2.7 \mathrm{~Hz}, 3 \mathrm{H}) \mathrm{ppm} ;{ }^{13} \mathrm{C}$ NMR $(75.4 \mathrm{MHz}$, $\left.\mathrm{CDCl}_{3}\right) \delta 218.6,197.5,136.8,133.5,130.6,130.4,128.2$, $126.9,126.5,126.1,125.3,123.9,104.8,78.2,13.8$ ppm; MS $(\mathrm{m} / \mathrm{z}) 208\left(\mathrm{M}^{+}, 63.16\right), 155$ (100); IR (neat) 3059, 1957, 1930, $1650,1508,1285,1251,1204,1155,1080,1059 \mathrm{~cm}^{-1}$; HRMSEI $(m / z)$ calcd for $\mathrm{C}_{15} \mathrm{H}_{12} \mathrm{O}\left[\mathrm{M}^{+}\right]$: 208.0888; found: 208.0887 .

\section{2-Allyl-1-phenylbuta-2,3-dien-1-one (2i)}

The reaction of 1,10-phenanthroline (5.4 mg, $0.03 \mathrm{mmol}), 2,2^{\prime}-$ bipyridine (4.6 mg, $0.03 \mathrm{mmol}), \mathrm{CuCl}(6.1 \mathrm{mg}, 0.06 \mathrm{mmol})$, $\mathrm{K}_{2} \mathrm{CO}_{3}(21.5 \mathrm{mg}, 0.15 \mathrm{mmol})$, dry toluene $(1.5 \mathrm{~mL})$, DBAD (13.7 mg, $0.06 \mathrm{mmol})$, and 2-allyl-1-phenylbuta-2,3-dien-1-ol $(55.1 \mathrm{mg}, 0.3 \mathrm{mmol}) /$ dry toluene $(1.5 \mathrm{~mL})$ afforded $\mathbf{2 i}(41.1 \mathrm{mg}$, $75 \%$ ) (eluent: petroleum ether/diethyl ether $=40: 1)$ : liquid; ${ }^{1} \mathrm{H}$ NMR (300 MHz, $\left.\mathrm{CDCl}_{3}\right) \delta 7.78(\mathrm{~d}, J=7.8 \mathrm{~Hz}, 2 \mathrm{H}), 7.50(\mathrm{t}$, $J=7.2 \mathrm{~Hz}, 1 \mathrm{H}), 7.39(\mathrm{t}, J=7.5 \mathrm{~Hz}, 2 \mathrm{H}), 5.98-5.82(\mathrm{~m}, 1 \mathrm{H})$, 5.22-5.04 (m, 4H), 3.20-3.14 (m, 2H) ppm; ${ }^{13} \mathrm{C} \mathrm{NMR}$ $\left(75.4 \mathrm{MHz}, \mathrm{CDCl}_{3}\right) \delta 217.2,194.1,138.0,134.9,132.1,129.0$, 127.8, 116.4, 105.3, 79.8, 32.5 ppm; MS (m/z) $184\left(\mathrm{M}^{+}, 2.53\right)$, 105 (100); IR (neat) 3081, 3062, 2982, 1956, 1931, 1651, 1598, $1578,1447,1422,1316,1272 \mathrm{~cm}^{-1}$; HRMS-EI $(\mathrm{m} / \mathrm{z})$ calcd for $\mathrm{C}_{13} \mathrm{H}_{12} \mathrm{O}\left[\mathrm{M}^{+}\right]$: 184.0888 ; found: 184.0889 . 


\section{2-Butyl-1-phenylnona-2,3-dien-1-one (2j)}

The reaction of 1,10-phenanthroline ( $5.6 \mathrm{mg}, 0.03 \mathrm{mmol}), 2,2^{\prime}$ bipyridine (4.9 mg, $0.03 \mathrm{mmol}), \mathrm{CuCl}(6.2 \mathrm{mg}, 0.06 \mathrm{mmol})$, $\mathrm{K}_{2} \mathrm{CO}_{3}$ (21.5 mg, $\left.0.15 \mathrm{mmol}\right)$, dry toluene $(1.5 \mathrm{~mL})$, DBAD (14.1 mg, $0.06 \mathrm{mmol}$ ), and 2-butyl-1-phenylnona-2,3-dien-1-ol $(82.2 \mathrm{mg}, 0.3 \mathrm{mmol}) /$ dry toluene $(1.5 \mathrm{~mL})$ afforded $\mathbf{2 j}$ [43] (74.8 mg, 91\%): liquid; ${ }^{1} \mathrm{H}$ NMR (300 MHz, $\left.\mathrm{CDCl}_{3}\right) 7.71$ (d, $J=7.8 \mathrm{~Hz}, 2 \mathrm{H}), 7.50-7.42(\mathrm{~m}, 1 \mathrm{H}), 7.40-7.32(\mathrm{~m}, 2 \mathrm{H}), 5.36(\mathrm{t}$, $J=7.2 \mathrm{~Hz}, 1 \mathrm{H}), 2.48-2.30(\mathrm{~m}, 2 \mathrm{H}), 2.16-1.96(\mathrm{~m}, 2 \mathrm{H})$, $1.55-1.11(\mathrm{~m}, 10 \mathrm{H}), 0.93(\mathrm{t}, J=6.9 \mathrm{~Hz}, 3 \mathrm{H}), 0.84(\mathrm{t}, J=$ $7.2 \mathrm{~Hz}, 3 \mathrm{H}) \mathrm{ppm} ;{ }^{13} \mathrm{C} \mathrm{NMR}\left(75.4 \mathrm{MHz}, \mathrm{CDCl}_{3}\right) \delta 213.3,195.7$, 138.9, 131.5, 128.8, 127.6, 107.4, 95.0, 31.1, 30.2, 28.6, 28.4, $27.8,22.31,22.29,13.89,13.86 \mathrm{ppm}$.

\section{2-Propyl-1-phenylbuta-2,3-dien-1-one (2k)}

The reaction of 1,10-phenanthroline ( $49.1 \mathrm{mg}, 0.27 \mathrm{mmol}), 2,2^{\prime}-$ bipyridine (42.6 mg, $0.27 \mathrm{mmol}), \mathrm{CuCl}(54.2 \mathrm{mg}, 0.54 \mathrm{mmol})$, $\mathrm{K}_{2} \mathrm{CO}_{3}(373.8 \mathrm{mg}, 2.7 \mathrm{mmol})$, dry toluene $(9 \mathrm{~mL})$, DBAD (124.4 mg, $0.54 \mathrm{mmol}$ ), and 2-propyl-1-phenylbuta-2,3-dien-1ol $(1.0141 \mathrm{~g}, 5.4 \mathrm{mmol}) /$ dry toluene $(9 \mathrm{~mL})$ afforded $\mathbf{2 k}$ (0.7512 g, 74\%): liquid; ${ }^{1} \mathrm{H}$ NMR (300 MHz, $\left.\mathrm{CDCl}_{3}\right) \delta 7.76(\mathrm{~d}$, $J=7.5 \mathrm{~Hz}, 2 \mathrm{H}), 7.50(\mathrm{t}, J=7.4 \mathrm{~Hz}, 1 \mathrm{H}), 7.39$ (t, $J=7.5 \mathrm{~Hz}$, $2 \mathrm{H}), 5.05(\mathrm{~s}, 2 \mathrm{H}), 2.39(\mathrm{t}, J=7.2 \mathrm{~Hz}, 2 \mathrm{H}), 1.62-1.46(\mathrm{~m}, 2 \mathrm{H})$, 0.99 (t, $J=7.5 \mathrm{~Hz}, 3 \mathrm{H}) \mathrm{ppm} ;{ }^{13} \mathrm{C}$ NMR $\left(75.4 \mathrm{MHz}, \mathrm{CDCl}_{3}\right)$ $\delta 217.1,194.9,138.3,131.9,129.0,127.8,106.7,79.3,29.9$, 21.1, 13.7 ppm; MS (m/z) $186\left(\mathrm{M}^{+}, 6.46\right), 105$ (100); IR (neat) 2961, 2932, 2872, 1933, 1651, 1598, 1578, 1447, 1315, $1271 \mathrm{~cm}^{-1}$; HRMS-EI $(\mathrm{m} / \mathrm{z})$ calcd for $\mathrm{C}_{13} \mathrm{H}_{14} \mathrm{O}^{+}\left[\mathrm{M}^{+}\right]$: 186.1045; found: 186.1045 .

\section{General experimental procedure for the oxi- dation of allenic alcohols with pure oxygen 3-Hexylocta-1,2-dien-4-one (2I)}

Typical procedure: 1,10-phenanthroline $(6.9 \mathrm{mg}, 0.0375 \mathrm{mmol})$, 2,2'-bipyridine $(5.8 \mathrm{mg}, 0.0375 \mathrm{mmol}), \mathrm{CuCl}(5.9 \mathrm{mg}$, $0.06 \mathrm{mmol}$ ), and $\mathrm{K}_{2} \mathrm{CO}_{3}(20.9 \mathrm{mg}, 0.15 \mathrm{mmol})$ were added sequentially to an oven dried Schlenk tube, which was purged with air and refilled with oxygen (twice). Then $1.5 \mathrm{~mL}$ of dry toluene was added, the resulting mixture was stirred at $\mathrm{rt}$ for $0.5 \mathrm{~h}$ which was followed by the sequential addition of DBAD (14.0 mg, $0.06 \mathrm{mmol}$ ), 2-hexyl-1-butylbuta-2,3-dien-1-ol $(63.8 \mathrm{mg}, 0.3 \mathrm{mmol})$ and $1.5 \mathrm{~mL}$ of dry toluene. After stirring at $60{ }^{\circ} \mathrm{C}$ for $24 \mathrm{~h}$, the reaction mixture was filtered through silica gel (100-140 mesh) and washed with diethyl ether. Evaporation of the solvent and flash chromatography on silica gel (eluent: petroleum ether/ether $=50: 1)$ afforded $21(37.1 \mathrm{mg}$, $58 \%)($ conv. $=81 \%$, yield $=72 \%$ (based on the alcohol consumed)): liquid; ${ }^{1} \mathrm{H}$ NMR (300 MHz, $\left.\mathrm{CDCl}_{3}\right) \delta 5.14$ (d, $J=$ $2.7 \mathrm{~Hz}, 2 \mathrm{H}), 2.62$ (t, $J=7.4 \mathrm{~Hz}, 2 \mathrm{H}), 2.18-2.09(\mathrm{~m}, 2 \mathrm{H})$, $1.60-1.47(\mathrm{~m}, 2 \mathrm{H}), 1.42-1.18(\mathrm{~m}, 10 \mathrm{H}), 0.92-0.78(\mathrm{~m}, 6 \mathrm{H})$ ppm; ${ }^{13} \mathrm{C}$ NMR $\left(75.4 \mathrm{MHz}, \mathrm{CDCl}_{3}\right) \delta 216.2,201.3,108.5,79.3$,
38.9, 31.6, 28.8, 27.8, 27.2, 26.2, 22.5, 22.3, 14.0, 13.8 ppm; MS (m/z) $208\left(\mathrm{M}^{+}, 1.00\right), 85$ (100); IR (neat) 2958, 2929, 2862, 1934, 1679, 1461, $1174 \mathrm{~cm}^{-1}$; HRMS-EI $(\mathrm{m} / z)$ calcd for $\mathrm{C}_{14} \mathrm{H}_{24} \mathrm{O}^{+}\left[\mathrm{M}^{+}\right]$: 208.1827; found: 208.1828 .

\section{4-Pentyl-1-phenylhexa-4,5-dien-3-one (2m)}

The reaction of 1,10-phenanthroline $(6.9 \mathrm{mg}, 0.0375 \mathrm{mmol})$, 2,2'-bipyridine (5.9 mg, $0.0375 \mathrm{mmol}), \mathrm{CuCl}(6.1 \mathrm{mg}$, $0.06 \mathrm{mmol}), \mathrm{K}_{2} \mathrm{CO}_{3}(21.4 \mathrm{mg}, 0.15 \mathrm{mmol})$, dry toluene (1.5 mL), DBAD (13.9 mg, $0.06 \mathrm{mmol})$, and 2-pentyl-1(phenylethyl)buta-2,3-dien-1-ol $(72.7 \mathrm{mg}, 0.3 \mathrm{mmol}) / \mathrm{dry}$ toluene $(1.5 \mathrm{~mL}$ ) afforded $\mathbf{2 m}(43.8 \mathrm{mg}, 60 \%)$ (conv. $=81 \%$, yield $=74 \%$ (based on the alcohol consumed)): liquid; ${ }^{1} \mathrm{H}$ NMR $\left(300 \mathrm{MHz}, \mathrm{CDCl}_{3}\right) \delta 7.31-7.22(\mathrm{~m}, 2 \mathrm{H}), 7.22-7.13(\mathrm{~m}, 3 \mathrm{H})$, $5.13(\mathrm{~s}, 2 \mathrm{H}), 3.02-2.85(\mathrm{~m}, 4 \mathrm{H}), 2.22-2.10(\mathrm{~m}, 2 \mathrm{H}), 1.45-1.20$ $(\mathrm{m}, 6 \mathrm{H}), 0.88(\mathrm{t}, J=6.5 \mathrm{~Hz}, 3 \mathrm{H}) \mathrm{ppm} ;{ }^{13} \mathrm{C} \mathrm{NMR}(75.4 \mathrm{MHz}$, $\left.\mathrm{CDCl}_{3}\right) \delta 216.3,200.1,141.3,128.3,126.0,108.6,79.7,40.9$, $31.4,30.9,27.4,26.1,22.4,14.0 \mathrm{ppm}$; MS $(\mathrm{m} / \mathrm{z}) 242\left(\mathrm{M}^{+}\right.$, 0.87), 105 (100); IR (neat) 2956, 2928, 2861, 1933, 1678, 1496, 1456, 1171, $1100 \mathrm{~cm}^{-1}$; Anal. calcd for $\mathrm{C}_{17} \mathrm{H}_{22} \mathrm{O}: \mathrm{C}, 84.25 ; \mathrm{H}$, 9.15. found: C, 84.16; H, 9.50.

\section{Supporting Information}

\section{Supporting Information File 1}

${ }^{1} \mathrm{H}$ and ${ }^{13} \mathrm{C}$ NMR spectra of products prepared.

[http://www.beilstein-journals.org/bjoc/content/ supplementary/1860-5397-7-51-S1.pdf]

\section{Acknowledgements}

Financial support from the National Basic Research Program of China (2009CB825300) is greatly appreciated.

\section{References}

1. Sheldon, R. A.; Kochi, J. K. Metal-Catalyzed Oxidations of Organic Compounds; Academic Press: New York, 1981.

2. Trost, B. M.; Fleming, I.; Ley, S. V. Comprehensive Organic Synthesis; Pergamon: Oxford, 1991; Vol. 7.

3. Fatiadi, A. J. Synthesis 1976, 65. doi:10.1055/s-1976-23961

4. Cainelli, G.; Cardillo, G. Chromium Oxidations in Organic Chemistry; Springer: Berlin, 1984

5. Anastas, P.; Warner, J. Green Cemistry: Theory and Practice; Oxford University Press: Oxford, 1998.

6. Blackburn, T. F.; Schwartz, J. J. Chem. Soc., Chem. Commun. 1977, 157. doi:10.1039/C39770000157

7. Peterson, K. P.; Larock, R. C. J. Org. Chem. 1998, 63, 3185. doi:10.1021/jo971268k

8. Nishimura, T.; Onoue, T.; Ohe, K.; Uemura, S. J. Org. Chem. 1999, 64, 6750. doi:10.1021/jo9906734

9. ten Brink, G. J.; Arends, I. W. C. E.; Sheldon, R. A. Science 2000, 287, 1636. doi:10.1126/science.287.5458.1636 
10. Muzart, J. Tetrahedron 2003, 59, 5789. doi:10.1016/S0040-4020(03)00866-4

For a review.

11. Semmelhack, M. F.; Schmid, C. R.; Cortés, D. A.; Chou, C. S. J. Am. Chem. Soc. 1984, 106, 3374. doi:10.1021/ja00323a064

12. Markó, I. E.; Giles, P. R.; Tsukazaki, M.; Brown, S. M.; Urch, C. J. Science 1996, 274, 2044. doi:10.1126/science.274.5295.2044

13. Markó, I. E.; Gautier, A.; Dumeunier, R.; Doda, K.; Philippart, F.; Brown, S. M.; Urch, C. J. Angew. Chem., Int. Ed. 2004, 43, 1588 doi:10.1002/anie.200353458

14. Sheldon, R. A.; Arends, I. W. C. E.; ten Brink, G. J.; Dijksman, A. Acc. Chem. Res. 2002, 35, 774. doi:10.1021/ar010075n

15. Zhan, B.; Thompson, A. Tetrahedron 2004, 60, 2917. doi:10.1016/j.tet.2004.01.043

16. Marshall, J. A.; Robinson, E. D. J. Org. Chem. 1990, 55, 3450. doi:10.1021/jo00298a012

17. Mashall, J. A.; Wang, X. J. Org. Chem. 1991, 56, 960. doi:10.1021/jo00003a013

18. Hashmi, A. S. K. Angew. Chem., Int. Ed. 1995, 34, 1581. doi:10.1002/anie.199515811

19. Hashmi, A. S. K.; Schwarz, L.; Choi, J. H.; Frost, T. M. Angew. Chem., Int. Ed. 2000, 39, 2285. doi:10.1002/1521-3773(20000703)39:13<2285::AID-ANIE2285>3.0.CO ;2-F

20. Ma, S.; Zhang, J. Chem. Commun. 2000, 117. doi:10.1039/a908627g

21. Ma, S.; Yin, S.; Li, L.; Tao, F. Org. Lett. 2002, 4, 505. doi:10.1021/ol0170859

22. Ma, S.; Yu, Z. Angew. Chem., Int. Ed. 2002, 41, 1775. doi:10.1002/1521-3773(20020517)41:10<1775::AID-ANIE1775>3.0.CO ;2-8

23. Ma, S.; Zhang, J.; Lu, L. Chem.-Eur. J. 2003, 9, 2447. doi:10.1002/chem.200305341

24. Ma, S.; Yu, S.; Yin, S. J. Org. Chem. 2003, 68, 8996. doi:10.1021/jo034633i

25. Ma, S.; Yu, Z. Chem.-Eur. J. 2004, 10, 2078. doi:10.1002/chem.200305341

26. Ma, S.; Yu, S. Org. Lett. 2005, 7, 5063. doi:10.1021/ol052073z

27. Ma, S.; Gu, Z.; Yu, Z. J. Org. Chem. 2005, 70, 6291. doi:10.1021/jo0507441

28. Sromek, A. W.; Rubina, M.; Gevorgyan, V. J. Am. Chem. Soc. 2005, 127, 10500. doi:10.1021/ja053290y

29. Dudnik, A. S.; Gevorgyan, V. Angew. Chem., Int. Ed. 2007, 46, 5195. doi:10.1002/anie.200701128

30. Marx, V. M.; Burnell, D. J. Org. Lett. 2009, 11, 1229. doi:10.1021/ol900029d

31. Alcaide, B.; Almendros, P.; Martínez del Campo, T. Eur. J. Org. Chem. 2007, 2844. doi:10.1002/ejoc.200700128

32. Sampath, M.; Loh, T.-P. Chem. Commun. 2009, 1568. doi:10.1039/b819959k

33. Zhao, J.; Loh, T.-P. Angew. Chem., Int. Ed. 2009, 48, 7232. doi:10.1002/anie.200902471

34. Russell, S. W.; Weedon, B. C. L. J. Chem. Soc. D 1969, 85. doi:10.1039/C29690000085

35. Landor, P. D.; Landor, S. R.; Mukasa, S. J. Chem. Soc. D 1971, 1638. doi:10.1039/C29710001638

36. Rannulu, N. S.; Rodgers, M. T. J. Phys. Chem. A 2007, 111, 3465. doi:10.1021/jp066903h

37. Reetz, M. T.; Li, X. Angew. Chem., Int. Ed. 2005, 44, 2962. doi:10.1002/anie.200462613
38. Reetz, M. T. Angew. Chem., Int. Ed. 2008, 47, 2556. doi:10.1002/anie.200704327

39. Li, J.; Zhou, C.; Fu, C.; Ma, S. Tetrahedron 2009, 65, 3695. doi:10.1016/j.tet.2009.02.061

40. Mukaiyama, T.; Harada, T. Chem. Lett. 1981, 10, 621. doi:10.1246/cl.1981.621

41. Alcaide, B.; Almendros, P.; Aragoncillo, C.; Redondo, M. C. Eur. J. Org. Chem. 2005, 98. doi:10.1002/ejoc.200400527

42. Yang, Y.; Shen, Z.; Loh, T.-P. Org. Lett. 2009, 11, 1209. doi:10.1021/ol8027362

43. Isaac, M. B.; Chan, T. H. J. Chem. Soc., Chem. Commun. 1995, 1003. doi:10.1039/C39950001003

44. Ma, S.; Yu, S.; Yin, S. J. Org. Chem. 2003, 68, 8996. doi:10.1021/jo034633i

\section{License and Terms}

This is an Open Access article under the terms of the Creative Commons Attribution License

(http://creativecommons.org/licenses/by/2.0), which permits unrestricted use, distribution, and reproduction in any medium, provided the original work is properly cited.

The license is subject to the Beilstein Journal of Organic Chemistry terms and conditions: (http://www.beilstein-journals.org/bjoc)

The definitive version of this article is the electronic one which can be found at: doi:10.3762/bjoc. 7.51 